\title{
Expression profile of long non-coding RNAs in cardiomyocytes exposed to acute ischemic hypoxia
}

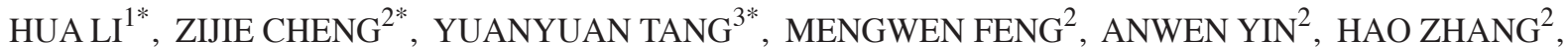 \\ JIA XU $^{2}$, QIJUN ZHANG $^{4}$, JINSONG ZHANG ${ }^{1}$ and LINGMEI QIAN ${ }^{2}$
}

\author{
Departments of ${ }^{1}$ Emergency and ${ }^{2}$ Cardiology, The First Affiliated Hospital of Nanjing Medical University, \\ Nanjing, Jiangsu 210029; ${ }^{3}$ Departmant of General Medicine, Traditional Chinese Medicine Hospital of Jiangsu Province, \\ Nanjing, Jiangsu 210004; ${ }^{4}$ Department of Cardiology, YinZhou Hospital Affiliated to Medical School of \\ Ningbo University, Ningbo, Zhejiang 315040, P.R. China
}

Received April 6, 2018; Accepted September 28, 2018

DOI: $10.3892 / \mathrm{mmr} .2018 .9658$

\begin{abstract}
Acute myocardial infarction (AMI) is a life-threatening disease and seriously influences patient quality of life. Long non-coding RNAs (lncRNAs), an emerging class of non-coding genes, have attracted attention in research, however, whether IncRNAs serve a function in acute ischemic hypoxia remains to be elucidated. In the present study, an IncRNA microarray was used to analyze differential lncRNA expression in acute ischemic hypoxia. A total of 323 lncRNAs were identified, 168 of which were upregulated and 155 of which were downregulated. Gene Ontology and Pathway analyses were also used to identify the potential functions of dysregulated lncRNAs; it was predicted that these dysregulated IncRNAs may contribute to the initiation of AMI. It was demonstrated that an IncRNA termed sloyfley may influence acute ischemic hypoxia through its neighboring gene Peg3, which has been linked to brain ischemia hypoxia. In summary, the present study identified numerous IncRNAs, which may provide further opportunities for the development of novel therapeutic strategies.
\end{abstract}

\section{Introduction}

Acute myocardial infarction (AMI) is a serious disease that affects numerous people around the world (1). The incidence

Correspondence to: Professor Lingmei Qian, Department of Cardiology, The First Affiliated Hospital of Nanjing Medical University, 300 Guangzhou Road, Nanjing, Jiangsu 210029, P.R. China E-mail: 1mqian@njmu.edu.cn

Professor Jinsong Zhang, Department of Emergency, The First Affiliated Hospital of Nanjing Medical University, 300 Guangzhou Road, Nanjing, Jiangsu 210029, P.R. China

E-mail: zhangjso@sina.com

*Contributed equally

Key words: long non-coding RNA, acute myocardial infarction, hypoxia of AMI is 208 cases per 100,000 a year (2). AMI is a life-threatening disease and seriously influences patient quality of life. Therefore, increased understanding of its pathogenesis may shed new light on novel diagnostic methods and active intervention.

The pathology of AMI mainly comprises persistent acute ischemic hypoxia caused by vascular stenosis and increased cardiac pressure. To date, investigations of ischemic hypoxia have focused on endoplasmic reticulum stress (ERS) (3), autophagy (4) and protein synthesis (5). Alleviating ERS can mitigate cardiac injury to a certain extent, however, the specific pathological mechanism underlying acute ischemic hypoxia remains to be elucidated.

Long non-coding RNAs (lncRNAs) are RNA molecules which are $>200$ nucleotides in length and have no coding potential. IncRNAs can be classified into intergenic lncRNAs, intronic lncRNAs, antisense lncRNAs, promoter-associated lncRNAs and UTR-associated lncRNAs. IncRNAs have been demonstrated to be associated with several diseases, including obesity (6), tumorigenesis (7) and congenital heart disease (8). lncRNAs serve significant functions, including structural or trafficking roles (9), cell differentiation and apoptosis (8). IncRNAs also function via a broad range of mechanisms, including regulating neighboring genes (10), microRNA-sponge action (11) and coding small peptides to suppress colon cancer (12). However, the lncRNA profile in neonatal rat cardiomyocytes exposed to ischemic hypoxia remains to be elucidated. There is no doubt that investigating the role and mechanism of lncRNAs in the pathophysiology of acute ischemic hypoxia will increase the understanding of AMI.

In the present study, a microarray profile was performed to identify differential lncRNA expression in cardiomyocytes. Altogether, 323 lncRNAs were identified, 168 of which were upregulated and 155 of which were downregulated. A total of 10 lncRNAs were randomly selected to verify the microarray results. It was predicted that these dysregulated lncRNAs may contribute to the process of AMI. Furthermore, an lncRNA termed sloyfley attracted attention due to its neighboring gene Peg3, which has been linked to brain ischemia hypoxia (13). Bioinformatics analysis was performed 
and the coding potential and possible interaction proteins and sequence of sloyfley were predicted. In summary, the findings provide comprehensive data regarding dysregulated lncRNAs in acute ischemic hypoxia and may provide further opportunities to assist in the development of therapeutic strategies.

\section{Materials and methods}

Cell culture. Neonatal male Sprague-Dawley rats (2-3 days old; 5-8 g; obtained from Nanjing Medical University) were immediately anaesthetized with $75 \%$ ethanol and their hearts were sheared and placed in cold PBS. The hearts were then digested using $0.4 \%$ type 2 collagenase $/ 0.6 \%$ pancreatin (Sigma-Aldrich; Merck KGaA, Darmstadt, Germany). Following digestion for 20 min, horse serum (HS; Sigma-Aldrich; Merck KGaA) was used to terminate the process and all the samples were centrifuged at $300 \mathrm{x} \mathrm{g}$ for $5 \mathrm{~min}$ at room temperature. This process was repeated until all the tissue had been completely digested. The cell pellets were resuspended in Dulbecco's modified Eagle's medium (DMEM) containing 5\% fetal bovine serum (FBS), 10\% HS and 1.2\% penicillin/streptomycin (all from Gibco; Thermo Fisher Scientific, Inc., Waltham, MA, USA). The neonatal rat cardiomyocytes were cultured in $5 \% \mathrm{CO}_{2}$ at $37^{\circ} \mathrm{C}$. The present study was approved by the Animal Care and Management Committee of Nanjing Medical University (Nanjing, China). Cell purity was evaluated by indirect immunofluorescence staining with a monoclonal anti-troponin T antibody under a fluorescence microscope (cat. no. ab92546; 1:2,000; Abcam, Cambridge, UK).

Acute ischemic hypoxia exposure. The neonatal rat cardiomyocytes were cultured for 4 days prior to exposure to acute ischemic hypoxia. The cardiomyocytes were randomly assigned to two groups. The growth media of the ischemic hypoxia group was replaced by serum-free DMEM for $2 \mathrm{~h}$, following which the cells were placed in a hypoxic environment which was achieved using an AnaeroPack system anaerobic jar. The AnaeroPack system can deplete the concentration of oxygen to $<1 \%$ within $30 \mathrm{~min}$. The growth media of the ischemic hypoxia group was replaced with serum-free DMEM for $2 \mathrm{~h}$, and the cells were placed in the hypoxic environment produced using the AnaeroPack system anaerobic jar. The cardiomyocytes were placed in the hypoxic environment. Following $3 \mathrm{~h}$ in hypoxia, the cells well collected in TRIzol for further investigation.

Reactive oxygen species (ROS) detection. The cardiomyocytes were incubated with H2DCF-DA $(10 \mu \mathrm{mol} / \mathrm{l})$ for $30 \mathrm{~min}$ at $37^{\circ} \mathrm{C}$ and then rinsed with PBS three times. DCFH-DA is a probe which can enter cells to measure the ROS production (14). DCFH-DA is not fluorescent and can pass through the cell membrane freely. On entering the cell, it can be hydrolyzed by the intracellular esterase to form DCFH, making it easy for the probe to be loaded into the cell. Intracellular ROS can oxidize non-fluorescent DCFH to produce fluorescent DCF. Detecting DCF fluorescence can be used to measure the levels of intracellular ROS. The fluorescence was observed with a fluorescence microscope (BX61; Olympus Corporation, Tokyo, Japan).
Lactate dehydrogenase ( $L D H)$ detection. The cell supernatant was collected for LDH analysis. Destruction of the cell membrane structure caused by apoptosis or necrosis results in the release of enzymes in the cytoplasm into the culture medium, including LDH with stable enzyme activity (15). Under the action of LDH, nicotinamide adenine dinucleotide $\left(\mathrm{NAD}^{+}\right)$is reduced to produce NADH. NADH and 2-(4-iodoph enyl)-3-(4-nitrophenyl)-5-phenyl-2H-tetrazolium are catalyzed by lipoamide dehydrogenase to form $\mathrm{NAD}^{+}$and chromophorin, and an absorption peak is generated at a wavelength of $490 \mathrm{~nm}$; therefore, the activity of LDH can be quantified by colorimetry. The release of LDH was spectrophotometrically measured using the CytoTox 96 Non-Radioactive Cytotoxicity Assay kit (cat. no. G1780; Promega Corporation, Madison, WI, USA) according to the manufacturer's protocol.

Cell survival calculation. The cardiomyocytes were digested for $3 \mathrm{~min}$ and then pipetting completely. Trypan blue staining was used to assess the cell survival rates, under a light microscope.

Microarray analysis. Rat Transcriptome Assay 1.0 was designed for the global profiling of differential lncRNA in acute ischemic hypoxia in rat models, providing a rich data set sufficient to decipher gene expression changes. The lncRNAs were collected from Ensembl (asia.ensembl.org/index.html), RefSeq (www.ncbi.nlm.nih.gov/refseq), Aceview (www.ncbi. nlm.nih.gov/ieb/research/acembly), and NONCODE (www. noncode.org). Positive probes for housekeeping genes and negative probes were also printed onto the array for hybridization quality control. The microarray hybridization was performed according to the manufacturer's protocol.

Gene Ontology (GO) and pathway analysis. The Gene Ontology (http://www.geneontology.org) program is a cooperative effort to describe the requirement for gene products in different databases. The present study analyzed the biological processes that differential lncRNAs may contribute to; the data were analyzed based on GO terms. Fisher's exact test and the v2 test were used to classify the GO category. Pathway analysis is a functional analysis for mapping genes to Kyoto Encyclopedia of Genes and Genomes pathways (david.ncifcrf.gov). The lower the P-value, the more important the pathway.

Reverse transcription-quantitative polymerase chain reaction $(R T-q P C R)$ analysis. The National Center for Biotechnology Information (NCBI; www.ncbi.nlm.nih.gov/pubmed) was used to design the primers and they were synthesized by Generay Biotech Co., Ltd. (Shanghai, China) (Table I). Briefly, total RNA was extracted from cardiomyocytes using TRIzol reagent (Thermo Fisher Scientific, Inc.). RNA purity was determined by measuring the absorbance ratio at 260/280 nm using a NanoDrop ND-1000 spectrophotometer (Thermo Fisher Scientific, Inc.). RNA was reverse transcribed using a PrimeScript ${ }^{\mathrm{TM}}$ RT reagent kit with gDNA eraser (cat. no. RR047A; Takara Bio, Inc., Otsu, Japan) at $37^{\circ} \mathrm{C}$ for $15 \mathrm{~min}, 85^{\circ} \mathrm{C}$ for $5 \mathrm{sec}$ and $4^{\circ} \mathrm{C}$ for $5 \mathrm{~min}$. qPCR was performed using SYBR ${ }^{\circledR}$ Premix Ex Taq ${ }^{\mathrm{TM}}$ (cat. no. RR420A; Takara Bio, Inc.), using the following thermocycling conditions: $95^{\circ} \mathrm{C}$ for $30 \mathrm{sec}$, followed by 40 cycles of $95^{\circ} \mathrm{C}$ for $5 \mathrm{sec}$ and $60^{\circ} \mathrm{C}$ 
Table I. Primers used in the present study for quantitative polymerase chain reaction analysis.

\begin{tabular}{ll}
\hline Primer & \multicolumn{1}{c}{ Sequence (5'-3') } \\
\hline stodee & F: TGGAAAACGTTAGAGCCTGAGG \\
& R: CCCAAAGAAGGGGAGAAGCT \\
RGD735140 & F: GCATGAAGCACAAGAGGG \\
& R: AGTTTTCATCATTGAAGATG \\
smaler & F: TGCTCGCAGTGGACTCTCTGTTG \\
& R: TGCTCGCAGTGGACTCTCTGTTG \\
LOC102548059 & F: GAGAAGGAGTCAGATGGTAC \\
& R: GCGGCTTCTCAAAGCTGATC \\
XLOC028424 & F: TTTTTTGGGATTTGTATTGATTT \\
& R: AAAACTTACCACCTCTAATCCAC \\
sleyko & F: CAGAGAAGAAGGGCTGGGG \\
& R: GCCTCTCTTTTCACTGCTGGTTCA \\
dakla & F: CAGGCTTCAGGCTCTGCTTCC \\
& R: CCCACTCTTCGCCCAGTGCT \\
loydey & F: AGGGCTATTGAATCTCAGAGG \\
& R: GGAGATGACCCCTCCATCTCC \\
sleegler & F: CTGCAATGTTTAAGCATTACTA \\
& R: GCTGGCTGAAATTCCAAATTC \\
slorlar & F: GAGGTGTGGGGCCGCAGC \\
GAPDH & R: ACGCCCTCACTAGAATGT \\
& F: ACCACAGTCCATGCCATCAC \\
& R: TCCACCACCCTGTTGCTGTA \\
&
\end{tabular}

F, forward; R, reverse.

for $30 \mathrm{sec}$. Data were normalized to GAPDH levels and further analyzed by the $2^{-\Delta \Delta \mathrm{Cq}}$ method (16). All the primers used for qPCR were listed in Table I.

Bioinformatics analysis. The neighboring genes were detected using NCBI. In addition, the location of sloyfley was labeled in red. Coding potential was calculated using calculator analyses (Peking University, Beijing, China). To identify the potential proteins that may interact with the lncRNA sloyfley, catRAPID (service.tartaglialab. com/page/catrapid_group) was used to simulate the candidate proteins. Computational predictions of ribonucleoprotein complex structures facilitate the detection of protein-RNA interactions and their molecular function. The potential binding sequence was also supplied using the RNA-Binding Protein Database (rbpdb.ccbr.utoronto.ca/index.php), and the transcription factor motifs which combined with the lncRNA sloyfley were predicted.

Statistical analysis. SPSS 13.0 (SPSS, Inc., Chicago, IL, USA) was used to calculate all values. Values are presented as the mean \pm standard error of the mean. Statistical analyses were performed using Student's t-test. $\mathrm{P}<0.05$ was considered to indicate a statistically significant difference.

\section{Results}

Identification of differentially expressed IncRNAs. ROS and LDH were selected to verify the acute ischemic hypoxia model (17). Following hypoxia for $3 \mathrm{~h}$, ROS and LDH levels were significantly increased compared with levels in the control group (Fig. 1A and B). The cell survival rates were also measured using trypan staining. The cell survival rates in the hypoxia group were decreased compared with those in the control group (Fig. 1C), suggesting that the acute ischemic hypoxia model had been established successfully. The Affymetrix rat lncRNA microarray was designed for the global profiling of lncRNA and protein-coding transcripts. The differentially expressed lncRNAs were carefully selected from the Ensembl, RefSeq RatGenome, AceView, NONCODE and lncRNA database (Fig. 1D). Scatter plots were used for visualization and assessment of the variation of lncRNA expression (Fig. 1E). By setting the threshold for differential expression at $\mathrm{P}<0.05$ and a fold change of $\geq 1.5$, a total of 323 IncRNAs were identified, 168 of which were upregulated and 155 of which were downregulated.

Expression signatures of differentially expressed lncRNAs. Following strident selection, the general signatures of distinctly expressed lncRNAs, including length distribution and chromosome distribution, were summarized. The lncRNAs were mainly 400-800 nucleotides in length (Fig. 2A). Chromosomal distribution showed the numbers of dysregulated lncRNAs located on different chromosomes (Fig. 2B).

GO and pathway analyses. GO analysis accessible online (http://www.geneontology.org). GO analysis was performed in the present study to discern the possible biological processes in which the lncRNAs may be involved. The genes potentially relevant were as follows: i) GO:2001244, positive regulation of intrinsic apoptotic signaling pathway; ii) GO:0042771, intrinsic apoptotic signaling pathway in response to DNA damage by p53 class mediator; iii) GO:0070059, intrinsic apoptotic signaling pathway in response to endoplasmic reticulum stress; iv) GO:0008625, extrinsic apoptotic signaling pathway via death domain receptors; v) GO:0097190, apoptotic signaling pathway (Fig. 3A). Of note, the biological processes acquired were relevant to the apoptotic signaling pathway to a large extent. The apoptotic signaling pathway has been investigated extensively, particularly the intrinsic pathway. However, whether lncRNAs can influence cell death through the apoptotic signaling pathway remains to be elucidated.

To further characterize the functional significance of the differentially expressed IncRNAs, pathway analysis was performed and the following five enriched pathways were identified: i) MAPK signaling pathway; ii) Apoptosis; iii) PI3K-Akt signaling pathway; iv) Hedgehog signaling pathway; v) Metabolic pathways (Fig. 3B). Based on this computed signaling network, it was found that multiple signaling procedures, including apoptosis and the MAPK signaling pathway, were significant to the network.

Confirmation of candidate lncRNAs. To validate the microarray results, five upregulated lncRNAs (stodee, RGD735140, smaler, LOC102548059 and XLOC_028424) 


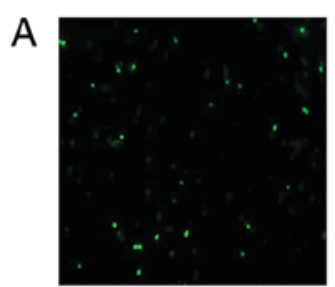

Control

D

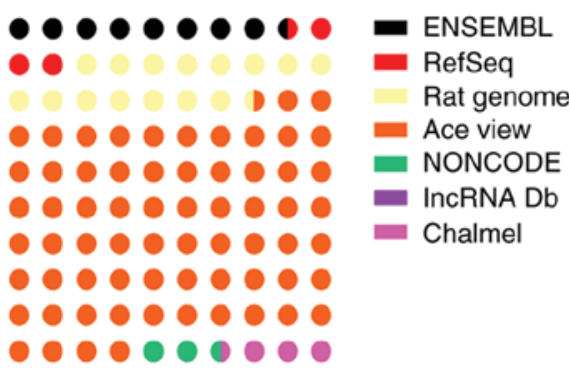

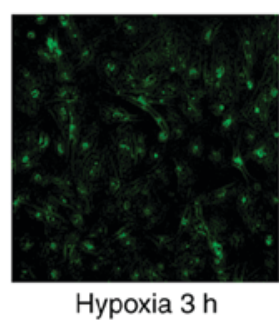
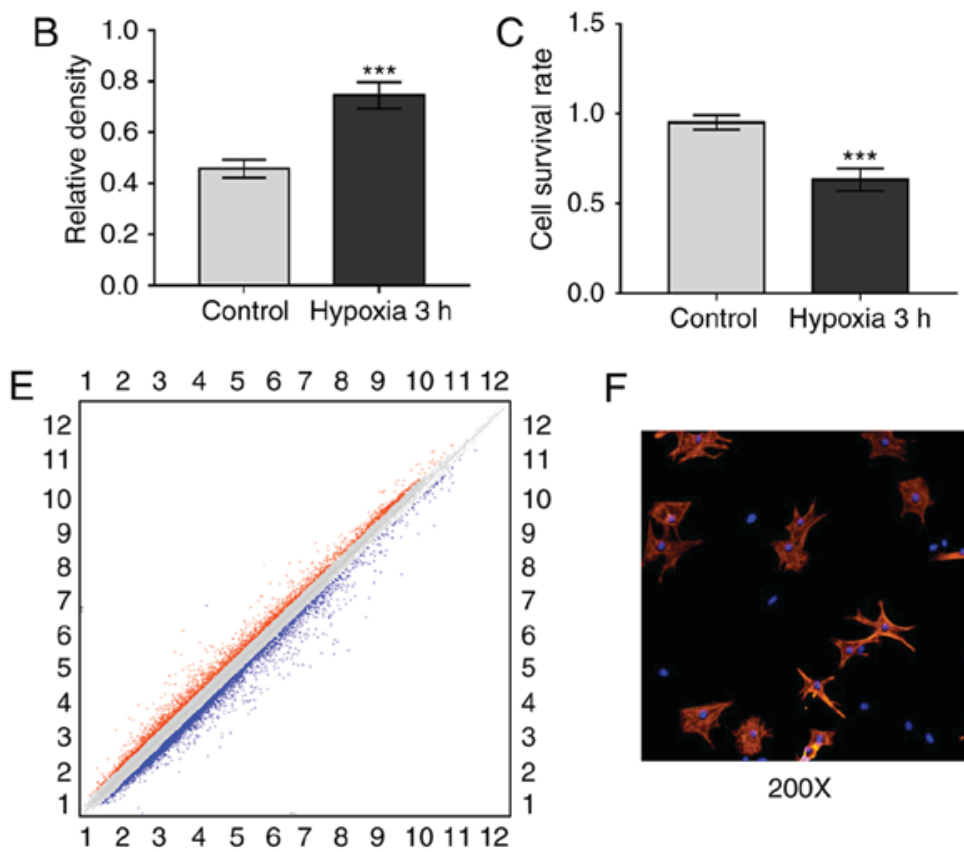

F

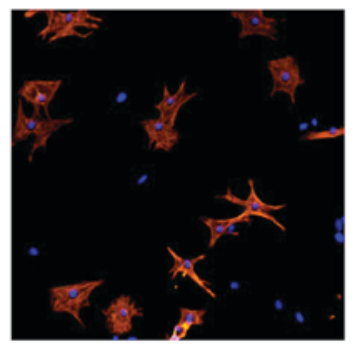

$200 x$

Figure 1. Acute ischemic hypoxia model and microarray profile. (A) Reactive oxygen species in the acute ischemic hypoxia group was significantly increased compared with that in the control group (magnification, x100). (B) Lactate dehydrogenase was increased in the acute ischemic hypoxia group compared with that in the control group. (C) Cell survival rate in the acute ischemic hypoxia group was decreased compared with that in the acute ischemic hypoxia group. (D) Pie chart of the relative numbers of differential lncRNAs. (E) Scatter plot demonstrating the differential lncRNAs. The numbers indicate the relative expression of differential lncRNAs. (F) Cardiomyocyte purity was identified using immunofluorescence staining with anti-troponin $\mathrm{T}$ antibody.

${ }^{* * * *} \mathrm{P}<0.001$ vs. control group (Student's t-test). lncRNAs, long non-coding RNAs.

and five downregulated lncR NAs (sleyko, dakla, Loydey, sleegler and slorlar) were randomly selected for verification. The five lncRNAs selected were upregulated compared with the control group (Fig. 4A).The downregulated lncRNAs were also verified using RT-qPCR analysis and the five lncRNAs were downregulated compared with the control group (Fig. 4B). Therefore, a series of lncRNAs were acquired that were constantly differentially expressed in acute ischemic hypoxia.

Bioinformatics and coding potential of sloyfley. Non-coding genes can be located within the intronic area and can overlap with the sense gene or antisense gene. The coding pattern is important in the function and mechanism of a candidate lncRNA. Differing locations often decide the fate of lncRNAs, which may function through the cis-mechanism or trans-mechanism. Among the dysregulated lncRNAs, a candidate was selected with a relatively high expression and an associated neighboring gene with possible functions in apoptosis and relevant pathways. An lncRNA named sloyfley attracted attention due to its high expression level and its neighboring gene Peg3 (Fig. 5A), which has been demonstrated to contribute to the process of brain ischemic hypoxia (13). The coding potential of IncRNA sloyfley was the calculated using Coding Potential Calculator analyses (Peking University, Beijing, China). Sloyfley had no credible protein-coding open reading frame with a coding potential score of 1.21214 (Fig. 5B). To further identify the potential protein that may interact with lncRNA sloyfley, catRAPID analysis was performed to calculate the interaction with potential protein ELAVL2 (Fig. 5C). The prediction of transcription factor binding sites indicated the possible interaction sequence (Fig. 5D).

\section{Discussion}

In the present study, differential lncRNAs were identified in cardiomyocytes exposed to acute ischemic hypoxia. GO and Pathway analyses were performed to assess the potential function of dysregulated lncRNA. Analysis was also performed of the bioinformatics information for lncRNA sloyfley, with neighboring gene Peg3, which has previously been demonstrated to be associated with brain ischemic hypoxia. The relative potential binding sequence of sloyfley was also identified. The findings of the present study provided comprehensive data regarding dysregulated lncRNAs in acute ischemic hypoxia, and may provide further opportunities for therapeutic strategies.

lncRNAs are known to regulate the expression of neighboring genes through a cis-mechanism (18), or to function through a distinct location instead of the transcription location as a trans-mechanism (9). In previous years, thousands of lncRNAs have been identified in numerous diseases (19). A persistent challenge in the investigation of lncRNAs is identifying the association between candidate lncRNAs and neighboring genes (20). To examine the function of candidate lncRNAs, the function of adjacent genes is an important element of the investigation. One possible mechanism is that lncRNAs may act locally, regulating the expression of neighboring gene transcripts (21). In the present study, the distinct lncRNA expression in cardiomyocytes exposed to acute ischemic hypoxia was investigated. It was predicted that the dysregulated lncRNAs may contribute to acute myocardial infarction. It was demonstrated that sloyfley may influence acute ischemic hypoxia, and the neighboring gene Peg3 has been demonstrated to be associated with brain ischemic 

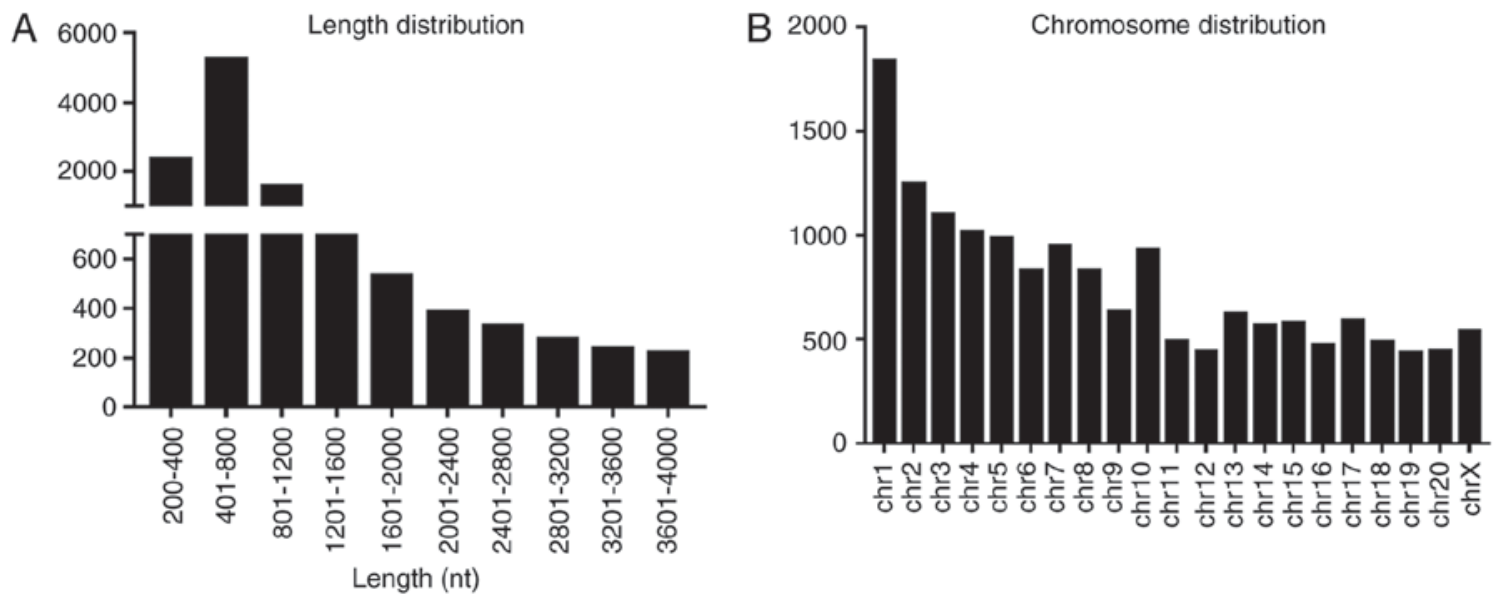

Figure 2. Signatures of differentially expressed lncRNAs. (A) Length distribution of differentially expressed lncRNAs. (B) Chromosome distribution of differentially expressed lncRNAs. lncRNAs, long non-coding RNAs; chr, chromosome.

A

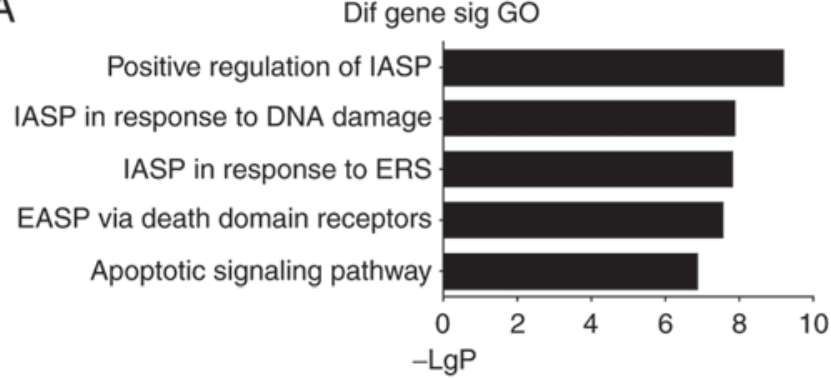

B

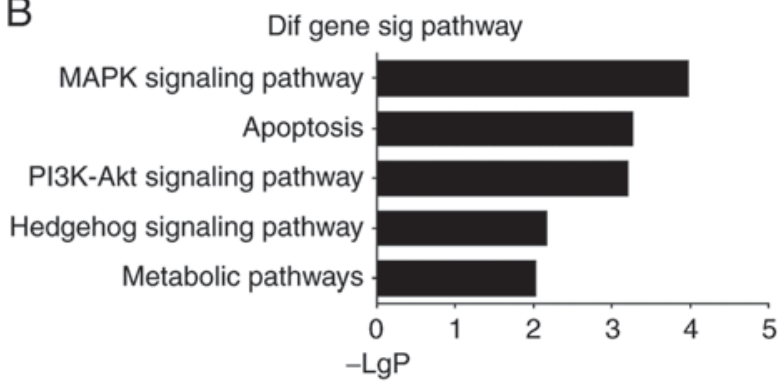

Figure 3. GO and Pathway analyses. (A) Top five GO terms. (B) Top five pathways. GO, Gene Ontology; Dif, differential; Sig, significant.
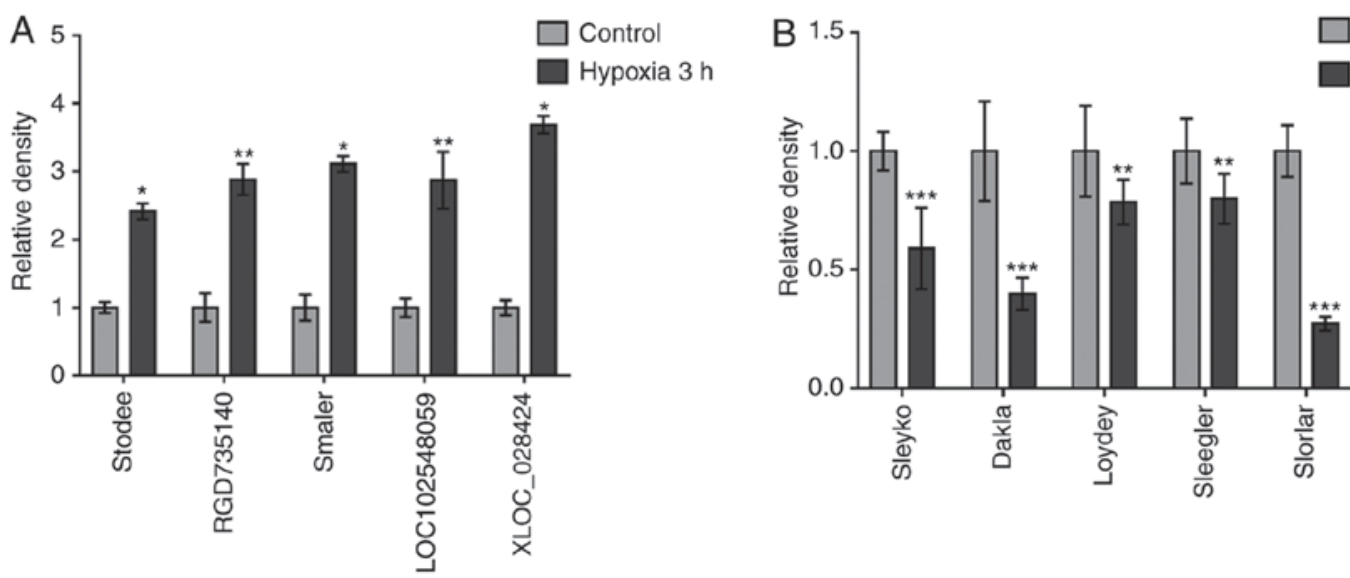

Figure 4. Validation of microarray results. (A) Five upregulated lncRNAs were randomly selected using RT-qPCR analysis. (B) Five downregulated lncRNAs were randomly selected using RT-qPCR analysis. ${ }^{*} \mathrm{P}<0.05,{ }^{* * *} \mathrm{P}<0.01,{ }^{* * *} \mathrm{P}<0.001$ vs. control group (Student's t-test). lncRNAs, long non-coding RNAs; RT-qPCR, reverse transcription-quantitative polymerase chain reaction.

hypoxia. Peg3 is involved in the p53-mediated cell death pathway as a downstream effector of p53 in brain ischemia hypoxia (13). However, the association between sloyfley and Peg3 remains to be elucidated. The results of the present study showed the potential binding sequence of sloyfley; specific experiments of the potential mechanism and its effect in acute ischemic hypoxia are clearly warranted.

GO and Pathway analyses were performed to identify the potential functions to which IncRNA may contribute (22). The results revealed that the dysregulated IncRNA was associated with the apoptosis process. The apoptotic pathway can be classified into two pathways (23); the extrinsic pathway involves cell surface receptors, whereas the intrinsic pathway utilizes the mitochondria activity and endoplasmic reticulum. The two pathways lead to the activation of caspases. Apoptosis is important in the pathophysiology of different diseases (24). Inadequate, excessive or limited apoptosis may all contribute to the pathogenesis of disease $(25,26)$. Perhaps the current 


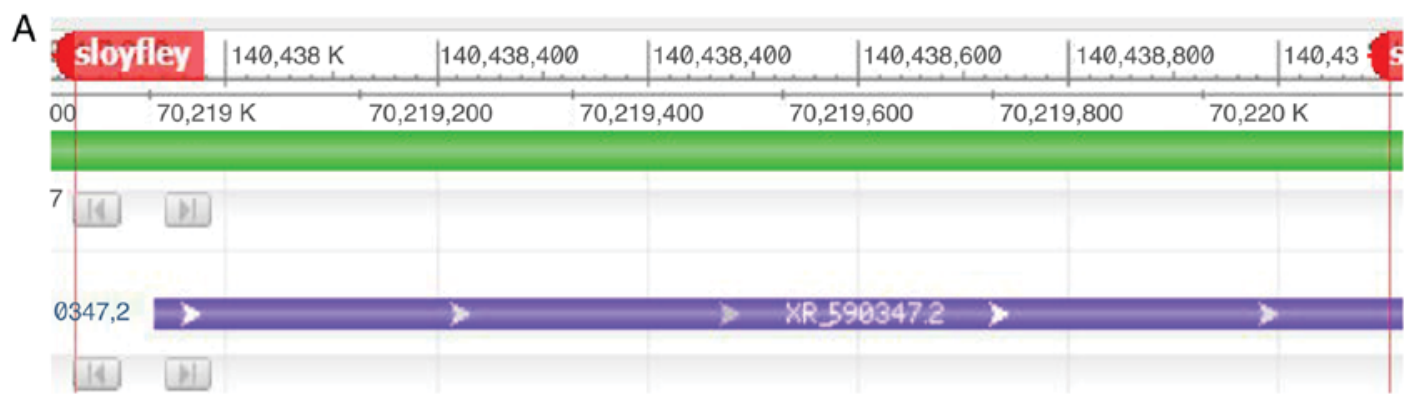

\begin{tabular}{|l|l|l|l|}
\hline \multirow{4}{*}{ HOMOLOGY FEATURES } & HIT NUM & 0 & \\
\cline { 2 - 4 } & HIT SCORE & 0.0 & \\
\hline & FRAME SCORE & 0.0 & \\
\hline \multirow{2}{*}{ ORF_FRAMEFINDER } & COVERAGE & $12.01 \%$ & \\
\hline & LOG-ODDS SCORE & 27.89 & \\
\hline & TYPE & Full & \\
\hline
\end{tabular}

C Legend: non-coding Coding

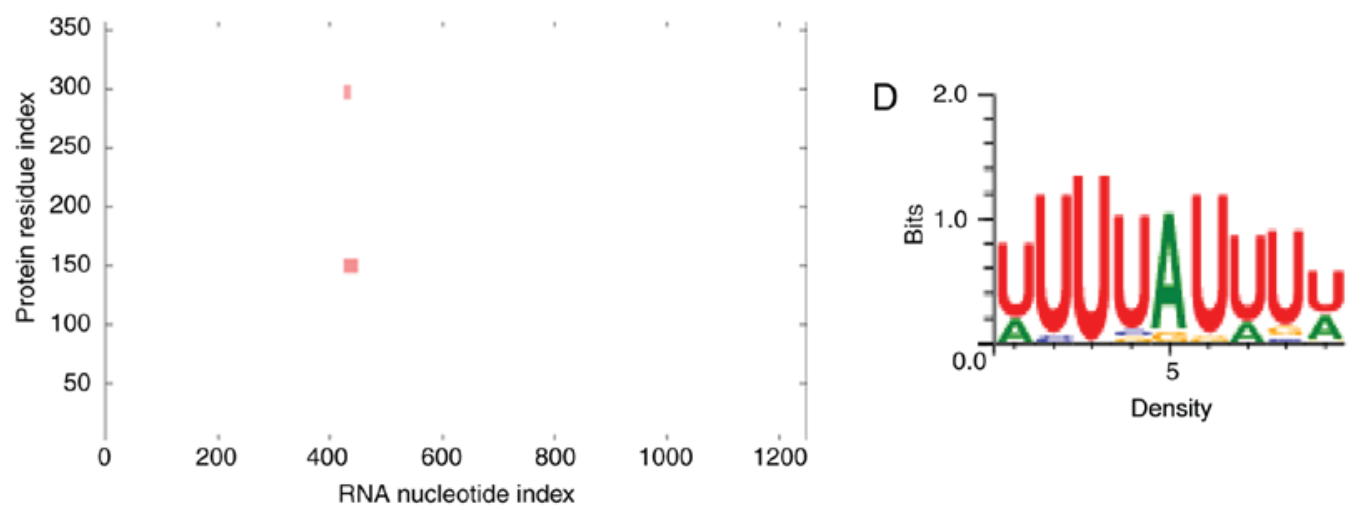

Figure 5. Bioinformatics analysis of sloyfley. (A) Location of sloyfley was detected using the National Center for Biotechnology Information. (B) Coding potential of sloyfley was calculated; no ORF was detected. (C) catRAPID analysis demonstrated a strong interaction between sloyfley and ELAVL2. (D) Predicted binding sequence of sloyfley. Density represents the relative binding potential. ORF, open reading frame.

challenge in investigating lncRNAs is the knowledge embedded in pathways regarding how various IncRNAs interact with their target genes. The results of the present study demonstrated that the biological processes of the dysregulated lncRNA may contribute to the apoptotic pathway. The PI3K-Akt pathway regulates several cellular processes, including cell apoptosis (27), ERS (28) and autophagy (29). Apoptosis is also involved in acute ischemic hypoxia (30). Whether IncRNA regulates acute ischemic hypoxia through the apoptotic pathway remains to be fully elucidated and further investigations are required in order to elucidate this. Of note, although the remaining lncRNAs were not selected for further analysis, this does not mean that they have no biological relevance. One possible explanation that may account for this is that lncRNAs may utilize different mechanisms to function, depending on their location.

In conclusion, the present study is the first, to the best of our knowledge, to present comprehensive data regarding the differential profile of IncRNAs in acute ischemic hypoxia. The findings suggest that numerous lncRNAs are involved in AMI and provide adequate resources for the future functional investigation of lncRNAs. There is no doubt that examining the role and mechanism of lncRNAs in the pathophysiology of acute ischemic hypoxia will provide further knowledge regarding AMI.

\section{Acknowledgements}

Not applicable.

\section{Funding}

This study was supported by the National Natural Science Foundation of China (grant nos. 81570209, 81873540 and 81800283).

\section{Availability of data and materials}

All data generated or analyzed during this study are included in this published article.

\section{Authors' contributions}

LQ, JZ and HL designed and supervised the study. HL, YT, and JX performed the experiments. $\mathrm{ZC}$ acquired the data. MF, AY, QZ and HZ analyzed the data. ZC and HL wrote 
the manuscript. All authors read and approved the final manuscript.

\section{Ethics approval and consent to participate}

Not applicable.

\section{Patient consent for publication}

Not applicable.

\section{Competing interests}

The authors declare that they have no competing interests.

\section{References}

1. Li YF, Wang H, Fan Y, Shi HJ, Wang QM, Chen BR, Khurwolah MR, Long QQ, Wang SB, Wang ZM and Wang LS: Epigallocatechin-3-gallate inhibits matrix metalloproteinase-9 and monocyte chemotactic protein-1 expression through the 67- $\mathrm{KDa}$ laminin receptor and the TLR4/MAPK/NF- $\kappa \mathrm{B}$ signalling pathway in lipopolysaccharide-induced macrophages. Cell Physiol Biochem 43: 926-936, 2017.

2. Lyngbakken MN, Skranes JB, De Lemos JA, Nygård S, Dalen H, Hveem K, Røsjø H and Omland T: Impact of smoking on circulating cardiac troponin I concentrations and cardiovascular events in the general population: The HUNT study (Nord-Trøndelag Health Study). Circulation 134: 1962-1972, 2016

3. Mccarroll CS, He W, Foote K, Bradley A, Mcglynn K, Vidler F, Nixon C, Nather K, Fattah C, Riddell AH, et al: Runx1 deficiency protects against adverse cardiac remodeling following myocardial infarction. Circulation 137: 57-70, 2018.

4. Maejima Y, Kyoi S, Zhai P, Liu T, Li H, Ivessa A, Sciarretta S, Del Re DP, Zablocki DK, Hsu CP, et al: Mst1 inhibits autophagy by promoting the interaction between Beclin1 and Bcl-2. Nat Med 19: 1478-1488, 2013

5. Simon MC, Liu L, Barnhart BC and Young RM: Hypoxia-induced signaling in the cardiovascular system. Annu Rev Physiol 70: $51-71,2008$.

6. Cui X, You L, Li Y, Zhu L, Zhang F, Xie K, Cao Y, Ji C and Guo X: A transcribed ultraconserved noncoding RNA, uc.417, serves as a negative regulator of brown adipose tissue thermogenesis. FASEB J 30: 4301-4312, 2016.

7. Liu X, Dai C, Jia G, Xu S, Fu Z, Xu J, Li Q, Ruan H and Xu P. Microarray analysis reveals differentially expressed lncRNAs in benign epithelial ovarian cysts and normal ovaries. Oncol Rep 38 799-808, 2017.

8. Song G, Shen Y, Ruan Z, Li X, Chen Y, Yuan W, Ding X, Zhu L and Qian L: lncRNA-uc.167 influences cell proliferation, apoptosis and differentiation of P19 cells by regulating Mef2c. Gene 590: 97-108, 2016.

9. Wang P, Xu J, Wang Y and Cao X: An interferon-independent lncRNA promotes viral replication by modulating cellular metabolism. Science 358: 1051-1055, 2017.

10. Joung J, Engreitz JM, Konermann S, Abudayyeh OO, Verdine VK, Aguet F, Gootenberg JS, Sanjana NE, Wright JB, Fulco CP, et al: Genome-scale activation screen identifies a lncRNA locus regulating a gene neighbourhood. Nature 548: 343-346, 2017.

11. Wang K, Liu F, Zhou LY, Long B, Yuan SM, Wang Y, Liu CY, Sun T, Zhang XJ and Li PF: The long noncoding RNA CHRF regulates cardiac hypertrophy by targeting miR-489. Circ Res 114: 1377-1388, 2014.
12. Huang JZ, Chen M, Chen, Gao XC, Zhu S, Huang H, Hu M, Zhu $\mathrm{H}$ and Yan GR: A peptide encoded by a putative lncRNA HOXB-AS3 suppresses colon cancer growth. Mol Cell 68 171-184.e6, 2017.

13. Yamaguchi A, Taniguchi $\mathrm{M}$, Hori O, Ogawa $\mathrm{S}$, Tojo $\mathrm{N}$, Matsuoka N, Miyake S, Kasai K, Sugimoto H, Tamatani M, et al: $\mathrm{Peg} 3 / \mathrm{Pw} 1$ is involved in p53-mediated cell death pathway in brain ischemia/hypoxia. J Biol Chem 277: 623-629, 2002.

14. Zhang C, Liu K, Yao K, Reddy K, Zhang Y, Fu Y, Yang G, Zykova TA, Shin SH, Li H, et al: HOI-02 induces apoptosis and G2-M arrest in esophageal cancer mediated by ROS. Cell Death Dis 6: e1912, 2015.

15. Chen Y, Liu J, Zheng Y, Wang J, Wang Z, Gu S, Tan J, Jing Q and Yang $\mathrm{H}$ : Uncoupling protein 3 mediates $\mathrm{H}_{2} \mathrm{O}_{2}$ preconditioning-afforded cardioprotection through the inhibition of MPTP opening. Cardiovasc Res 105: 192-202, 2015.

16. Livak KJ and Schmittgen TD: Analysis of relative gene expression data using real-time quantitative PCR and the 2(-Delta Delta C(T)) method. Methods 25: 402-408, 2001

17. Liu H, Jing X, Dong A, Bai B and Wang H: Overexpression of TIMP3 protects against cardiac ischemia/reperfusion injury by inhibiting myocardial apoptosis through ROS/mapks pathway. Cell Physiol Biochem 44: 1011-1023, 2017.

18. Zhang Y and Cao X: Long noncoding RNAs in innate immunity. Cell Mol Immunol 13: 138-147, 2016.

19. Quinn JJ and Chang HY: Unique features of long non-coding RNA biogenesis and function. Nat Rev Genet 17: 47-62, 2016.

20. Hosono Y, Niknafs YS, Prensner JR, Iyer MK, Dhanasekaran SM, Mehra R, Pitchiaya S, Tien J, Escara-Wilke J, Poliakov A, et al: Oncogenic role of THOR, a conserved cancer/testis long non-coding RNA. Cell 171: 1559-1572.e20, 2017.

21. Luo S, Lu JY, Liu L, Yin Y, Chen C, Han X, Wu B, Xu R, Liu W, Yan $\mathrm{P}$, et al: Divergent lncRNAs regulate gene expression and lineage differentiation in pluripotent cells. Cell Stem Cell 18: 637-652, 2016.

22. Li X, Wu LJ, Gu M, Chen YM, Zhang QJ, Li H, Cheng ZJ, Hu P, Han SP, Yu ZB and Qian LM: Peptidomic analysis of amniotic fluid for identification of putative bioactive peptides in ventricular septal defect. Cell Physiol Biochem 38: 1999-2014, 2016.

23. Whelan RS, Kaplinskiy V and Kitsis RN: Cell death in the pathogenesis of heart disease: Mechanisms and significance. Annu Rev Physiol 72: 19-44, 2010.

24. Nagata $S$ and Tanaka M: Programmed cell death and the immune system. Nat Rev Immunol 17: 333-340, 2017.

25. Hetz C and Saxena S: ER stress and the unfolded protein response in neurodegeneration. Nat Rev Neurol 13: 477-491, 2017.

26. Ashkenazi A, Fairbrother WJ, Leverson JD and Souers AJ: From basic apoptosis discoveries to advanced selective BCL-2 family inhibitors. Nat Rev Drug Discov 16: 273-284, 2017.

27. Ho L, Tan SY, Wee S, Wu Y, Tan SJ, Ramakrishna NB, Chng SC, Nama S, Sczerbinska I, Chan YS, et al: ELABELA Is an endogenous growth factor that sustains hESC self-renewal via the PI3K/AKT pathway. Cell Stem Cell 17: 435-447, 2015.

28. Zeng L, Xiao Q, Chen M, Margariti A, Martin D, Ivetic A, Xu H, Mason J, Wang W, Cockerill G, et al: Vascular endothelial cell growth-activated XBP1 splicing in endothelial cells is crucial for angiogenesis. Circulation 127: 1712-1722, 2013.

29. Nikoletopoulou V, Sidiropoulou K, Kallergi E, Dalezios Y and Tavernarakis N: Modulation of autophagy by BDNF underlies synaptic plasticity. Cell Metab 26: 230-242.e5, 2017.

30. Flevaris P, Khan SS, Eren M, Schuldt AJ, Shah SJ, Lee DC, Gupta S, Shapiro A, Burridge P, Ghosh AK, et al: Plasminogen activator inhibitor type I controls cardiomyocytetransforming growth factor- $\beta$ and cardiac fibrosis. Circulation 136: 664-679, 2017.

This work is licensed under a Creative Commons Attribution-NonCommercial-NoDerivatives 4.0 International (CC BY-NC-ND 4.0) License. 